\title{
Effect of applications of collagen ointments on the healing of purulent-necrotic ulcers of finger tissues in cows
}

\author{
Victor Andenko ${ }^{1 *}$, Vladimir Tolkachev ${ }^{2 *}$, Sergey Kolomiytsev ${ }^{3 *}$ \\ ${ }^{1}$ Kursk State Agricultural Academy named after I. I. Ivanov, 70 Karl Marx St., Kursk Russian Federation
}

\begin{abstract}
The purpose of the research was to obtain new experimental samples of collagen antiseptic ointments and to evaluate the effect of their use on the healing of purulent-necrotic ulcers of the finger tissues in cows. To achieve this goal, two experimental samples of collagen ointment were made: the first sample was a collagen analog of «Levomekol» ointment, and the second sample was a collagen ointment based on «Kemistep+» hoof sanitizer. The evaluation of the effect of their application on the healing of purulent-necrotic ulcers of the finger tissues was carried out in two experimental groups of sick animals with purulent-necrotic ulcers of the hoof crumb tissues of 7 heads each. In the first experimental group of animals, the first experimental sample of the ointment was applied, in the second - the second experimental sample. According to the results of scientific studies, it was found that the use of collagen ointment with the disinfectant «Kemistep+» on purulent-necrotic ulcers of crumb contributed to a decrease in their number. The epithelization index in the second experimental group compared to the first experimental group indicated a more positive effect of the use of collagen ointment with the disinfectant «Kemistep+» on the healing of purulent-necrotic ulcers of the finger tissues in cows than similar applications of the collagen analogue of the ointment «Levomekol».
\end{abstract}

\section{Introduction}

Currently, there are serious problems in dairy cattle breeding that hinder the intensification of the industry, which consist in the massive spread of surgical limb diseases in lactating livestock [1]. Among all surgical diseases in cattle in the conditions of industrial dairy complexes, purulent-necrotic ulcerative pathologies in the tissues of the fingers have a significant degree of spread [2]. Purulent-necrotic ulcerative lesions of finger tissues in cows in the conditions of industrial dairy complexes cause significant economic damage resulted due to reduced milk production of sick animals, their culling and treatment costs [3]. In addition, the widespread and inappropriate use of various antibacterial ointments increases the resistance of pathogens of purulent surgical infection, complicating the healing process of purulent-necrotic tissue ulcers and increasing the recovery time of sick animals [4]. These circumstances dictate the need to search for and develop new multicomponent ointments with a wide range of antiseptic properties. effects without the content of antibiotics in their composition and capable of highly intensive stimulation of regeneration and epithelialization processes [5]. In this regard, it was considered relevant to produce new experimental samples of collagen antiseptic ointments and evaluate the effect of their applications on the healing of purulentnecrotic ulcers of finger tissues in cows by planimetric indicators of their area and epithelialization index, which served as the purpose of scientific research.

\section{Materials and methods}

Clinical and experimental studies were performed at the Department of Surgery and Therapy of the Kursk State Agricultural Academy, as well as in the production conditions of the dairy cattle breeding complex of JSC «Educational and Experimental Farm «Znamenskoye». At the beginning of the research, an orthopedic medical examination of the cattle population was conducted for the prevalence of purulent-necrotic ulcers of finger tissues, their localization and nosological specific structure, taking into account the technology of animal husbandry. By establishing the prevalence, localization, and nosological profilepurulent-necrotic ulcers of finger tissues in cows, diseased animals were divided into two experimental groups of 7 heads each according to the principle of analogues and treated with applications of the collagen analog of «Levomekol» ointment and collagen ointment with the disinfectant «Kemistep+». The first experimental sample of the ointment was made in the conditions of the Department of Surgery and Therapy of the Kursk State Agricultural Academy in the following sequence: initially, $100.0 \mathrm{~g}$ of pure chemical collagen was weighed into a glass flask and melted in a water bath; then into a collagen flask. The base was added $4.0 \mathrm{~g}$ of methyluracil and $0.75 \mathrm{~g}$ of levomycetin; after that, a prototype of the «Levomecol» analog 
ointment on a collagen basis was slowly cooled. The second prototype of a collagen ointment with the disinfectant «Kemistep+» was made according to the following technology: $100.0 \mathrm{~g}$ of pure chemical collagen was placed in a dry glass flask and melted in a water bath; $15.0 \mathrm{ml}$ of a solution of a concentrate of the hoof disinfectant «Kemistep+» and $4.0 \mathrm{~g}$ of methyluracil were added to the molten collagen; further, a molten collagen base with the disinfectant was added gradually cooled. During the experiment, the first prototype of the «Levomekol» analog ointment based on collagen was tested in the treatment of purulent-necrotic ulcers of finger tissues in cows in the first experimental group of animals, and the second prototype of the collagen ointment with the disinfectant «Kemistep+» was tested in the second experimental group of sick animals. To assess the effect of applications of collagen ointments on the healing of purulent-necrotic ulcers of finger tissues in cows, planimetric studies of their area were performed on the $3 \mathrm{rd}, 7$ th and 10 th days of therapy. Planimetric parameters studies included transferring the contours of ulcerative defects in finger tissues to millimeter paper, counting whole squares within the contour boundaries, and obtaining the area of ulcers in the appropriate accounting period. The obtained indicators of the planimetric area of purulent-necrotic ulcers were subjected to mathematical processing to calculate the epithelialization index $\Delta S(1)$ :

$$
\Delta \mathrm{S}=\frac{(S-S n) * 100}{\mathrm{~S} * \mathrm{t}}
$$

where $S$ is the area of the purulent-necrotic ulcer during the previous measurement; $\mathrm{Sn}$ is the area of the purulent-necrotic ulcer at the time of measurement in the corresponding accounting period; $\mathrm{t}$ - the number of days between the previous and subsequent measurements. Further, the digital indicators were subjected to statistical processing, with determination of the degree of reliability and comparative evaluation among themselves. On this basis, a conclusion was made about the effect of applications of various collagen ointments on the healing of purulent-necrotic ulcers of finger tissues in cows.

\section{Results and Discussion}

The results of orthopedic medical examination of cattle kept in the conditions of the dairy complex of JSC «Educational and Experimental Farm «Znamenskoye» indicated that out of 199 cows examined, 42 heads or $21.10 \%$ of the livestock showed the presence of purulent-necrotic ulcerative pathologies in the tissues of the distal part of the limbs. At the same time, it was found that out of 42 individuals of diseased animals, 18 heads or $42.85 \%$ were kept on a leash on a wooden floor, and 24 heads or $57.15 \%$ - without a leash on a concrete floor. Thus, the prevalence of purulent-necrotic ulcers of finger tissues in cows when keeping animals without a leash on a concrete floor was higher than in similar animals when kept on a leash on a wooden floor by $14.30 \%$ ( $\mathrm{p}<0.05)$, respectively.
Taking into account the localization of purulentnecrotic ulcers in the distal part of the limbs in cattle, showed that in $40.00 \%$ of the diseased patients or in 17 heads they were located in the area of the hoof crumb, in $28.00 \%$ of the examined animals or in 12 heads - in the area of the hoof corolla; in $20.00 \%$ or in 8 heads - in the area of the interdigital arch; in $12.00 \%$ or in 5 heads - in the area of the hoof sole. Detailed localization of the established nosological profile of purulent-necrotic ulcers of finger tissues in the thoracic and pelvic extremities indicated, that crumb and hoof corolla ulcers were more often located on the pelvic limbs. On the thoracic limbs, the greatest incidence of diagnosis of ulcers of the tissues of the interdigital hoof arch was recorded. Purulent-necrotic ulcers of the hoof sole had the same frequency of registration on both the thoracic and pelvic limbs.

In accordance with the results of orthopedic medical examination, it was considered necessary to treat the most common purulent-necrotic ulcerative pathology purulent - necrotic ulcers of the hoof crumb using experimental samples of collagen ointments and evaluate their effect on the dynamics of healing. Sick animals with purulent-necrotic crumb ulcers were divided according to the principle of analogues into two experimental groups of 7 heads each. In the first experimental group, applications of the collagen analog of «Levomekol» ointment were performed for ulcerative defects», and in the second - collagen ointment with the disinfectant «Kemistep+» and carried out the corresponding planimetric measurements of the area on the $3 \mathrm{rd}, 7 \mathrm{th}$ and 10th days of treatment.

The results of planimetric measurements of the area of purulent-necrotic ulcers of the hoof crumb tissues in the first experimental group indicated that on the 3rd day of applications, ulcerative defects had dimensions equal to $27.70 \pm 8.08 \mathrm{~mm}^{2}$. Thus, the area of ulcerative defects during three days of treatment with a collagen sample of «Levomekol» ointment decreased by $18.30 \pm 8.16 \mathrm{~mm}^{2}$ or by $39.78 \%$ ( $p<0.05)$. On the 7 th day of treatment in animals of the first experimental group, the planimetric dimensions of purulent-necrotic ulcers were 14.80 \pm 8.61 $\mathrm{mm}^{2}$ and were less than on the $3 \mathrm{rd}$ day of the experiment by $12.90 \pm 7.49 \mathrm{~mm}^{2}$ or by $46.57 \% \quad(\mathrm{p} \leq 0.05)$. In comparison with the initial size of ulcer defects before treatment, they decreased by $31.20 \pm 8.09 \mathrm{~mm}^{2}$ during the week of applying the collagen analog of «Levomekol» ointment or by $67.83 \%$ ( $p<0.05$ ). On the 10 th day of curation, the area of purulent-necrotic ulcers of the hoof crumb in cows of the first experimental group had a digital value equal to $9.40 \pm 4.74 \mathrm{~mm}^{2}$, which was less than on the 7 th day of planimetric measurements by $5.40 \pm 3.85 \mathrm{~mm}^{2}$ or by $36.49 \%$ ( $\mathrm{p}<0.05$ ), respectively. In addition, it was recorded that on the 10th day of applications of the collagen analog of «Levomekol» ointment for purulent-necrotic ulcerative defects, in cows of the first experimental group, their sizes were smaller than on the 3rd day of therapy by $18.30 \pm 6.47 \mathrm{~mm}^{2}$ or by $66.06 \%$ ( $\mathrm{p}<0.05$ ), and differed from the initial to therapeutic dimensions by $36.60 \pm 8.01 \mathrm{~mm}^{2}$ or by $79.56 \% \quad(\mathrm{p} \leq 0.05)$, respectively. Further planimetric calculations of the epithelialization index during 
applications for purulent-necrotic ulcerative defects of the hoof crumb tissues of the collagen analog of «Levomekol» ointment allowed us to establish that on the 3rd day of treatment it was $0.55 \pm 0.37$, on the 7 th day $-0.73 \pm 0.29$, on the 10 th day $-0.39 \pm 0.13$.

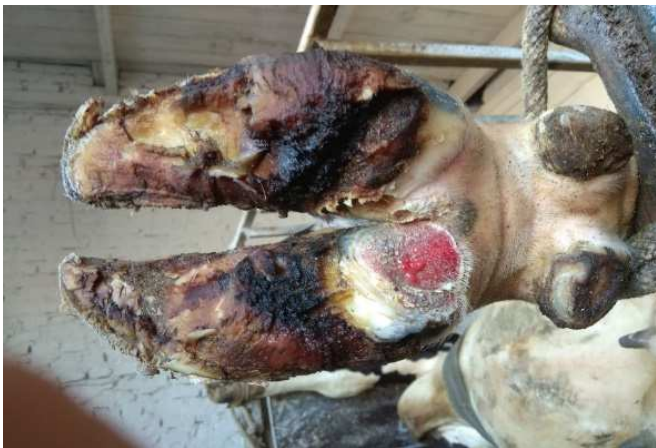

Fig. 1 Healing on the 3rd day of treatment with the collagen analogue of the ointment «Levomekol»

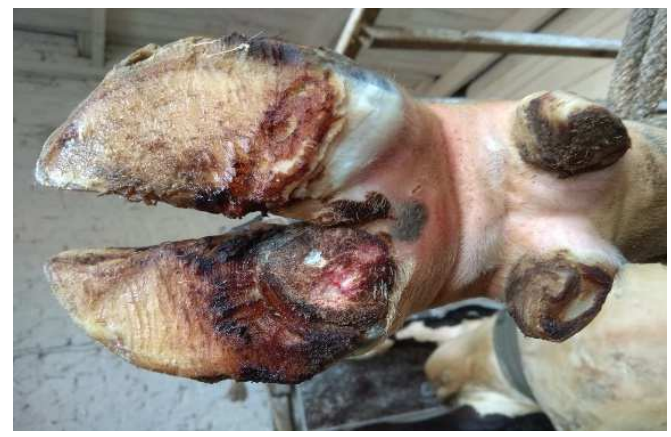

Fig. 2 Healing on the 7 th day of treatment with the collagen analogue of the ointment «Levomekol»

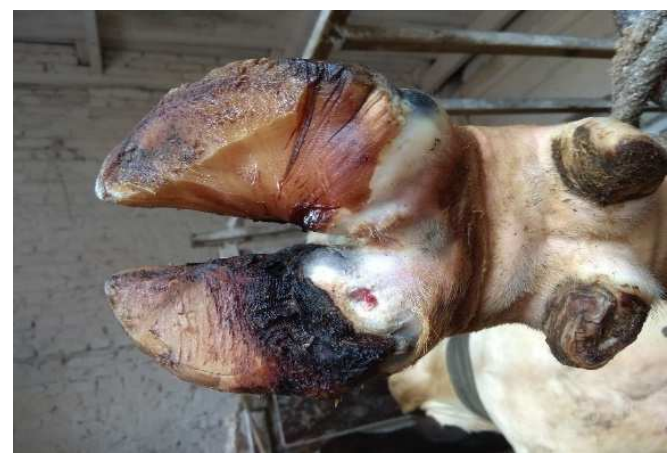

Fig. 3 Healing on the 10th day of treatment with the collagen analogue of the ointment «Levomekol»

Thus, the highest epithelialization index of ulcerative defects of the hoof crumb in cows of the first experimental group was recorded on the 7th day of treatment, which was higher than that on the $3 \mathrm{rd}$ and 10th days on the first experimental group $53.42 \%$ $(\mathrm{p} \leq 0.05)$ and $49.36 \%(\mathrm{p} \leq 0.05)$, respectively.

Treatment of animals of the second experimental group with applications of collagen ointment with the disinfectant «Kemistep+» on day 3 reduced the area of purulent-necrotic ulcerative defects of the hoof crumb by $22.20 \pm 8.04 \mathrm{~mm}^{2}$ up to dimensions of $23.80 \pm 8.03 \mathrm{~mm}^{2}$ or by $48.26 \%(\mathrm{p} \leq 0.05)$. On the 7 th day of treatment, the planimetric area of purulent-necrotic ulcers was $12.12 \pm 8.95 \mathrm{~mm}^{2}$.

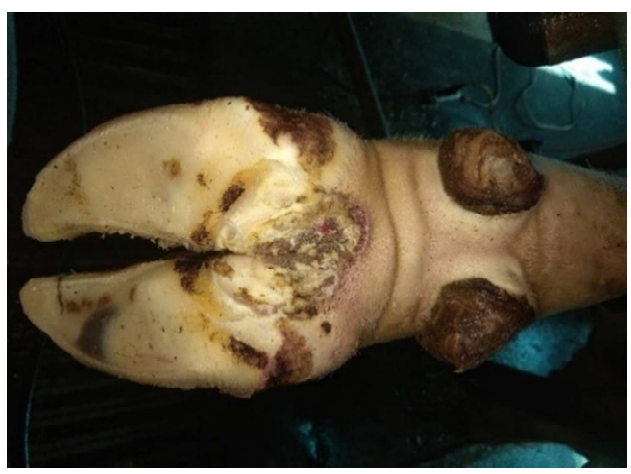

Fig. 4 Healing on the 3rd day of treatment with collagen ointment with the disinfectant «Kemistep+»

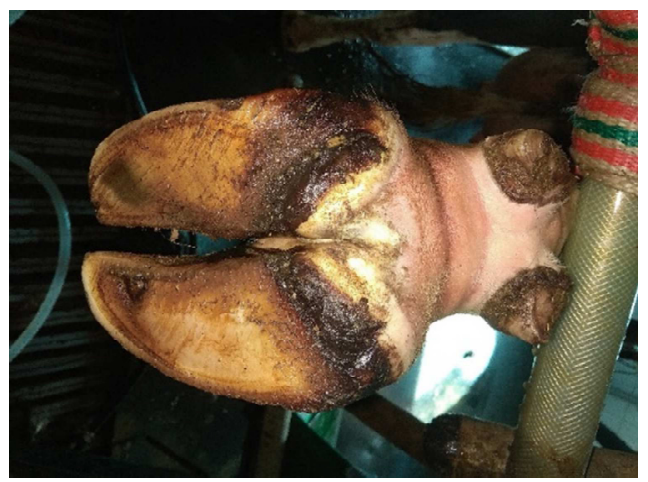

Fig. 5 Healing on the 7 th day of treatment with collagen ointment with the disinfectant «Kemistep+»

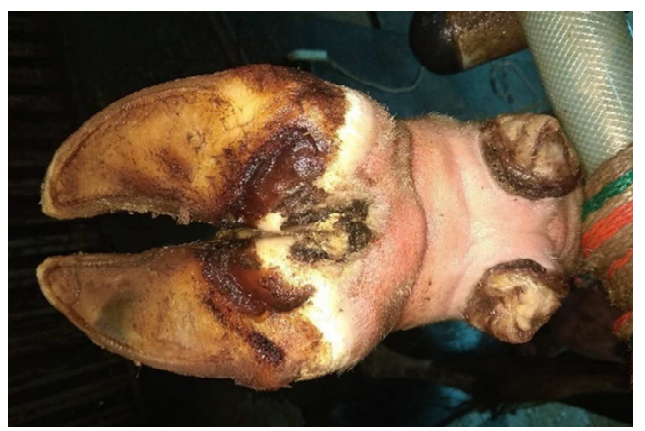

Fig. 6 Healing on the 10th day of treatment with collagen ointment with the disinfectant «Kemistep+»

Thus, during a week of applications of collagen ointment with the disinfectant «Kemistep+» for ulcerative defects, their area decreased from the initial to therapeutic size by $33.80 \pm 8.34 \mathrm{~mm}^{2}$ or by $73.49 \%$ $(\mathrm{p} \leq 0.05)$, and was also less than on the 3rd day of measurements by $11.60 \pm 8.02 \mathrm{~mm}^{2}$ or by $51.26 \%$ $(\mathrm{p} \leq 0.05)$, respectively. By the 10 th day of treatment of animals of the second experimental group, the area of purulent-necrotic ulcerative defects in the tissues of the hoof crumb had a digital expression equal to $9.20 \pm 1.42$ $\mathrm{mm}^{2}$. In comparison with the results of planimetric measurements of the area of hoof crumb ulcers on the 3rd and 7th days of applying collagen ointment with the disinfectant «Kemistep+», on the 10th day of the experiment, it was less by $14.60 \pm 6.48 \mathrm{~mm}^{2}$ or by $38.65 \%$ 
$(\mathrm{p} \leq 0.05)$ and by $3.00 \pm 4.12 \mathrm{~mm}^{2}$ or by $24.09 \%(\mathrm{p} \leq 0.05)$, respectively.

In addition, applications of collagen ointment with the disinfectant «Kemistep+» by the 10th day of treatment contributed to a reduction in the area of purulent-necrotic ulcers of finger tissues in cows of the second experimental group relative to the initial therapeutic size by $36.80 \pm 7.89 \mathrm{~mm}^{2}$ or by $80.00 \%$ $(\mathrm{p} \leq 0.05)$. Calculations of the epithelialization index of purulent-necrotic ulcers of hoof crumb tissues in cows of the second experimental group indicated that on day 3 it was $0.53 \pm 0.05$, on day $7-0.93 \pm 0.31$, on day $10-$ $0.83 \pm 0.23 \mathrm{~mm}^{2}$. In the comparative aspect, the highest epithelialization index of purulent-necrotic ulcerative defects in the tissues of the hoof crumb in sick animals during the application of collagen ointment with the disinfectant «Kemistep+» was noted on the 7th day of treatment and was higher than on the 3rd day by $56.98 \%$ $(\mathrm{p}<0.05)$ and on the 10th day by $10.75 \%(\mathrm{p}<0.05)$. Comparative analysis of the effect of applications of collagen ointments on the healing of purulent-necrotic ulcers of finger tissues in cows, showed that on the $3 \mathrm{rd}$ day, the area of hoof crumb ulcers in the second experimental group, when applying a collagen mask to the skin, was reduced. ointments with the disinfectant «Kemistep+» were less by $3.90 \pm 8.02 \mathrm{~mm}^{2}$ or by $14.08 \%$ $(\mathrm{p}<0.05)$ than in animals from the first experimental group when applying the collagen analog of «Levomekol» ointment to ulcerative surfaces. Similar dynamics were recorded in the subsequent terms of accounting for the planimetric area of ulcerative defects of the hoof crumb. Thus, on the 7th day of treatment, the area of purulent-necrotic tissue ulcers in sick cows from the second experimental group was less than in diseased animals from the first experimental group by $2.68 \pm 8.44$ $\mathrm{mm}^{2}$ or by $18.11 \%(\mathrm{p} \leq 0.05)$, and on the 10th day by $0.20 \pm 0.30 \mathrm{~mm}^{2}$ or by $3.13 \%(\mathrm{p}<0.05)$. A comparative assessment of the epithelialization index of purulentnecrotic ulcerative defects of hoof crumb tissues in cows of two experimental groups allowed us to establish that on the 7th and 10th days of applying collagen ointment with the disinfectant «Kemistep+» it was higher than when applying the collagen analog of «Levomekol» ointment by $21.50 \%(\mathrm{p}<0.05)$ and $53.01 \%(\mathrm{p}<0.05)$. It was found that the use of collagen ointment with the disinfectant «Kemistep+» had a more positive effect on the healing of purulent-necrotic tissue ulcers of the hoof crumb in cows, accelerating the dynamics of reduction of their planimetric area.

\section{Conclusion}

Performing scientific studies to assess the effect of applications of collagen ointments on the healing of purulent-necrotic ulcers of finger tissues in cows made it possible to establish that applications of the collagen analog of «Levomekol» ointment reduced their area by $39.78 \%(\mathrm{p} \leq 0.05)$ to the size of $27.70 \pm 8.08 \mathrm{~mm}-$ by 3 days of treatment, by the 7th day of treatment - by $46.57 \%(\mathrm{p} \leq 0.05)$ to the size of $14.80 \pm 8.61 \mathrm{~mm}^{2}$, by the 10th day of treatment - by $36.49 \%(\mathrm{p} \leq 0.05)$ to the size of $9.40 \pm 4.74 \mathrm{~mm}^{2}$. And the epithelialization index of ulcerative defects of hoof crumb tissues was equal to $0.55 \pm 0.37,0.73 \pm 0.29,0.39 \pm 0.13$, accordingly. Similar applications of collagen ointment with the disinfectant «Kemistep+» to purulent-necrotic ulcers of the finger tissues in cows reduced the planimetric dimensions of ulcerative defects in the hoof crumb tissues by $48.26 \%$ (p < 0.05 ) to $23.80 \pm 8.03 \mathrm{~mm}^{2}$ by day 3 of treatment, by day 7 of treatment by $51.26 \%(\mathrm{p} \leq 0.05)$ to values of $12.12 \pm 8.95 \mathrm{~mm}^{2}$, by the 10th day of treatment - by $24.09 \%(\mathrm{p} \leq 0.05)$ to values of $9.20 \pm 1.42 \mathrm{~mm}^{2}$. The epithelialization index of purulent-necrotic ulcers of hoof crumb tissues on day 3 of applications of collagen ointment with the disinfectant «Kemistep+» was $0.53 \pm 0.05$, on day $7-0.93 \pm 0.31$, on day $10-0.83 \pm 0.23$. A comparative analysis of the effect of applications of two experimental samples of collagen ointments on the healing of purulent - necrotic ulcers of finger tissues in cows indicated that on day 3 their planimetric dimensions in the treatment of collagen ointment with the disinfectant «Kemistep+» were less by $14.08 \%$ $(p \leq 0.05)$ than that in the application of collagen ointment analog «Levomekol», and on the 7th day and 10th day by $18.11 \%(\mathrm{p} \leq 0.05)$ and $3.13 \%(\mathrm{p} \leq 0.05)$, respectively. At the same time, it was determined that the epithelialization index of ulcerative defects of hoof crumb tissues with applications of collagen ointment with the disinfectant «Kemistep+» on the 7th and 10th days was higher by $21.50 \%(\mathrm{p} \leq 0.05)$ and $53.01 \%$ $(p \leq 0.05)$ than that with applications of collagen ointment analog «Levomekol». Thus, it was recorded that the application of collagen ointment with the disinfectant «Kemistep+» on purulent-necrotic ulcers of the hoof crumb tissues in experimental animals had the following effect: a more positive effect on the dynamics of healing than applications of the «Levomekol» analog collagen ointment, which more intensively reduces the planimetric dimensions of ulcerative defects and increases the epithelialization index of their surface.

\section{References}

1. K. Chiba, T. Miyazaki, Y. Sekiyama, M. Miyazaki, K. Okadata Vet. Med. Sci. 79 (7), 1191 - 1195, (2017)

2. G.T. Mamitov, A.A. Stekolnikov, V.A. Tolkachev, S.M. Kolomiytsev, M.A. Ladanova Regulatory issues in veterinary medicine. 4, 76 77, (2017)

3. A.N. Eliseev, A.A. Stepanov, V.A. Tolkachev Bulletin of the Kursk State Agricultural Academy. 1, $111-113,(2012)$

4. A.N. Eliseev, S.M. Kolomiytsev, A.I. Blednov, V.A. Tolkachev, D.N. Boldyrev, E.A. Steblovsky Bulletin of the Kursk State Agricultural Academy. 9, 71 - 74, (2013)

5. C. Bergsten Veter. Rec. 140(22), 574 - 577, (1997). 